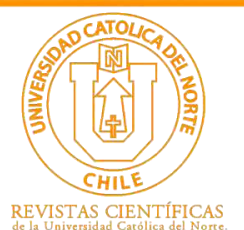

\title{
On additive maps of MA-semirings with involution
}

\author{
Liaqat Ali ${ }^{1}$ @ orcid.org/0000-0003-2352-1127 \\ Muhammad Aslam² (1) orcid.org/0000-0001-9429-625X \\ Yaqoub Ahmed Khan ${ }^{3}$ (0) orcid.org/0000-0001-9033-0916
}

Government College University, Dept. of Mathematics, Lahore, Pakistan.

1@ rehmani.pk786@gmail.com; ; ${ }^{2}$ aslam298@gcu.edu.pk; ${ }^{3}$ yaqoubahmedkhan@gmail.com

Received: February 2020 | Accepted: June 2020

\begin{abstract}
:
We extend the concept of *-derivations of rings to a certain class of semirings called MA-semirings and establish some results on commutativity forced by the *-derivations satisfying different criteria. We specially focus on the results on certain conditions under which additive mappings become Jordan *-derivations. -code, which, when self-dual, produces an unimodular lattice by Construction $A$.
\end{abstract}

Keywords: MA-semirings; *-semirings ; *-derivations; Jordan *- derivations.

MSC (2020): 16Y60, 16W10.

\section{Cite this article as (IEEE citation style):}

L. Ali, M. Aslam and Y. A. Khan, "On additive maps of MA-semirings with involution", Proyecciones (Antofagasta, On line), vol. 39 , no. 4, pp. 1097-1112, Aug. 2020, doi: 10.22199/issn.07176279-2020-04-0067.

Article copyright: (C) 2020 Liaqat Ali, Muhammad Aslam and Yaqoub Ahmed Khan. This is an open access article distributed under the terms of the Creative Commons License, which permits unrestricted use and distribution provided the original author and source are credited. 


\section{Introduction and Preliminaries}

The concept of involution is studied by many algebraists for algebras, groups, rings and other structures $[5,7,11,12,13,14,16]$. Another aspect which carries much importance in ring theory is derivation. M. Bresar and J. Vukman [6] studied the concept of *-derivation and Jordan *-derivation for rings. We can roughly say that a ${ }^{*}$-derivation is a derivation with involution. In the present paper, we canonically extend the concept of *derivation for a class of semirings called MA-semirings introduced by Javed et al [8]. For more on MA-semirings one can see [1, 2, 3, 4, 9, 15]. We generalize some results for *-derivations of MA-semirings established in [10] for rings.

Now we include some definitions and preliminaries necessary for completion. An additive inverse semiring $S$ with absorbing zero ' 0 ' is called an MA-Semiring if $r+r^{\prime} \in Z, \forall r \in S$, where $Z$ is the center of $S$ and $r^{\prime}$ is the pseudo inverse of $r$. Obviously every ring is an MA-semiring but the following example shows that converse may not be true.

Example 1.1. [8] The set $S=\{0,1,2,3,4, \ldots\}$ with addition $\oplus$ and multiplication $\odot$ respectively defined by $a \oplus b=\sup \{a, b\}$ and $a \odot b=\inf \{a, b\}$ is an $M A$-semiring. In fact $S$ is a commutative prime $M A$-semiring.

Such examples motivate us to generalize the results of ring theory for MAsemirings. Throughout the paper, by a ${ }^{*}$-semiring $S$, we mean a *-MAsemiring unless stated otherwise. $S$ is prime if $a R b=0$ implies that $a=0$ or $b=0 . S$ is semiprime if $a R a=0$ implies that $a=0$. An additive mapping $*: S \longrightarrow S$ is involution if $\forall u, v \in S,\left(u^{*}\right)^{*}=u$ and $(u v)^{*}=v^{*} u^{*}$. By a ${ }^{*}$-semiring we simply mean a semiring $S$ with involution $*$. Following example describes a *-MA-semiring.

Example 1.2. If $(R,+, \cdot)$ is an $M A$-Semiring, then the set $R$ with addition ' + ' and multiplication $\bullet$ defined as $a \bullet b=b$. $a$ forms an MA-semiring called the opposite MA-Semiring of $R$. We usually notate it as $R^{o}$.

Let $(R,+, \cdot)$ be an MA-semiring and $R^{o}$ its opposite MA-semiring. Consider $S=R \times R^{o}$ with $(a, b) \oplus(c, d)=(a+c, b+d)$ and $(a, b) \odot(c, d)=(a . c, b \bullet d)=$ $(a c, d b)$. Then $(S, \oplus, \odot)$ forms an MA-semiring. Define $*: S \rightarrow S$ by $(x, y)^{*}=(y, x)$. Therefore $*$ defines an involution on $S$ and $(S, \oplus, \odot)$ forms a $*$-MA-semiring or MA-semiring with involution.

$S$ is 2-torsion free if for $u \in S, 2 u=0$ implies $u=0$ and 3-torsion if $3 u=0$ implies $u=0$. An additive mapping $d: S \longrightarrow S$ is said to be a 
derivation if $d(u v)=d(u) v+u d(v)$. A *-derivation is an additive mapping $d: S \longrightarrow S$ such that $d(u v)=d(u) v^{*}+u d(v)$. By Jordan *-derivation, we mean an additive mapping $d: S \longrightarrow S$ satisfying $d\left(u^{2}\right)=d(u) u^{*}+u d(u)$. An additive mapping $F: S \rightarrow S$ is generalized derivation associated with a derivation $d$ if $F(x y)=F(x) y+x d(y)$. We define Commutator as $[u, v]=$ $u v+v^{\prime} u$. By Jordan product we mean $u \circ v=u v+y u$ for all $u, v \in S$. Following identities will be used frequently: $[u, u v]=u[u, v],[u v, w]=$ $u[v, w]+[u, w] v,[u, y w]=[u, v] w+v[u, w],[u, v]+[v, u]=v\left(u+u^{\prime}\right)=$ $u\left(v+v^{\prime}\right),(u v)^{\prime}=u^{\prime} v=u v^{\prime},[u, v]^{\prime}=\left[u, v^{\prime}\right]=\left[u^{\prime}, v\right], u \circ(v+w)=u \circ v+u \circ w$ (see $[8],[15])$.

\section{Main Results}

Theorem 2.1. Let $S$ be a semiprime ${ }^{*}$-semiring. If $T$ is an additive mapping satisfying

$$
T(u v)+T\left(u^{\prime}\right) v^{*}=0, \forall u, v \in S
$$

Then $[T(S), S]=0$ and hence $T(S) \subseteq Z(S)$.

Proof. In (2.1) writing $u w$ for $u$, we get $\forall u, v \in S$

$$
T(u w v)+T\left(u^{\prime} w\right) v^{*}=0
$$

and $w v$ for $v$ in $(2.2)$, we get $T(u w v)+T\left(u^{\prime}(w v)^{*}=0\right.$, which implies

$$
T(u w v)+T\left(u^{\prime}\right) v^{*} w^{*}=0
$$

From (2.3), put $T(u w v)=T(u w) v^{*}$, we get

$$
T(u w) v^{*}+T\left(u^{\prime}\right) v^{*} w^{*}=0
$$

From (2.1) using $T(u v)=T(u) v^{*}$ into (4), we obtain $T(u) w^{*} v^{*}+$ $T\left(u^{\prime}\right) v^{*} w^{*}=0$, which implies $T(u) w^{*} v^{*}+T(u)^{\prime} v^{*} w^{*}=0$ and therefore $T(u)\left(w^{*} v^{*}+v^{*^{\prime}} w^{*}\right)=0$, which further gives

$$
T(u)\left[w^{*}, v^{*}\right]=0
$$

In (2.5) replacing $w$ by $w^{*}$ and $v$ by $v^{*}$, we obtain

$$
T(u)[w, v]=0
$$


In (2.6), replacing $w$ by $w T(u)$, we obtain $T(u)[w T(u), v]=0$, which implies $T(u) w[T(u), v]+T(u)[w, v] T(u)=0$. Using (2.6) again, we obtain

$$
T(u) w[T(u), v]=0
$$

Replacing $w$ by $v w$ in (2.7), we obtain

$$
T(u) v w[T(u), v]=0
$$

Multiplying (2.7) by $v^{\prime}$ from the left, we obtain

$$
v^{\prime} T(u) w[T(u), v]=0
$$

Adding (2.8) and (2.9), we obtain $[T(u), v] w[T(u), v]=0$ which implies $[T(u), v] S[T(u), v]=0$. Since $S$ is semiprime, therefore the last equation yields $[T(u), v]=0$, which gives $[T(S), S]=0$ and hence $T(S) \subseteq Z(S)$.

Theorem 2.2. Let $S$ be prime ${ }^{*}$-semiring. If $S$ admits a nontrivial *derivation $d$ such that $d(u v)+d\left(u^{\prime}\right) d(v)=0, \forall u, v \in S$, then $d=0$.

Proof. By hypothesis for all $u, v \in S$, we have

$$
d(u v)+d\left(u^{\prime}\right) d(v)=0
$$

By definition of *-derivation,from (2.10), we obtain

$$
d(u) v^{*}+u d(v)+d\left(u^{\prime}\right) d(v)=0
$$

In (2.11) replacing $u$ by $u w$, we obtain $d(u w) v^{*}+u w d(v)+d\left(u^{\prime} w\right) d(v)=$ 0 and again using (2.11), we obtain $d(u) d(w) v^{*}+x w d(v)+d\left(u^{\prime} w\right) d(v)=0$, which after simplification implies $\left(d(u) d(w)+d\left(u^{\prime}\right) d(w)\right) v^{*}+\left(u+d\left(u^{\prime}\right)\right) w d(v)=$ 0 . Using (10), we obtain $\left(u+d\left(u^{\prime}\right)\right) w d(v)=0$ and therefore $\left(u+d\left(u^{\prime}\right)\right) S d(v)=$ 0 . As $S$ is prime, either $\left(u+d\left(u^{\prime}\right)\right)=0$ or $d(v)=0$. If $\left(u+d\left(u^{\prime}\right)\right)=0$, then $u=d(u)$, a contradiction, which shows that $d(v)=0$ and therefore $d=0$.

Theorem 2.3. Let $S$ be prime ${ }^{*}$-semiring. If $S$ admits a ${ }^{*}$-derivation $d$ such that $d \neq I^{*}$ and $d(u v)+d(v) d\left(u^{\prime}\right)=0, \forall u, v \in S$, then $d=0$ (where $\left.\left.I^{*}(u)=u^{*}\right)\right)$. 
Proof. By the hypothesis for all $u, v \in S$

$$
d(u, v)+d(v) d\left(u^{\prime}\right)=0
$$

In (2.12) writing $u v$ for $v$, we obtain $d(u u v)+d(u v) d\left(u^{\prime}\right)=0$ which further gives on simplification $d(u) v^{*}\left(u^{*}+d\left(u^{\prime}\right)\right)+u\left(d(u v)+d(v) d\left(u^{\prime}\right)\right)=$ 0 . Using (2.12) again, we obtain $d(u) v^{*}\left(u^{*}+d\left(u^{\prime}\right)\right)=0$, which implies $d(u) S\left(u^{*}+d\left(u^{\prime}\right)\right)=0$. By the primeness of $S$, we have either $u^{*}+d\left(u^{\prime}\right)=0$ or $d(u)=0$. If $u^{*}+d\left(u^{\prime}\right)=0$, then $d\left(u^{\prime}\right)=u^{*}=I^{*}(u)$, which implies that $d=I^{*}$, a contradiction. Therefore we obtain $d(u)=0$ and $d=0$ as required.

Theorem 2.4. Let $S$ be prime ${ }^{*}$-semiring and $a \in S$. If $S$ admits a *derivation $d$ such that $[d(u), a]=0 \forall u \in S$, then $a \in Z(S)$ or $d(a)=0$.

Proof. We have forall $u \in S$

$$
[d(u), a]=0
$$

In (13) replacing $u$ by $u v$, we obtain $[d(u v), a]=0$. On simplification, we obtain $d(u)\left[v^{*}, a\right]+[d(u), a] v^{*}+u[d(v), a]+[u, a] d(v)=0$. Using (13), again, we obtain

$$
d(u)\left[v^{*}, a\right]+[u, a] d(v)=0
$$

Replacing $u$ by $a$ in (2.14), we obtain $d(a)\left[v^{*}, a\right]+[a, a] d(v)=0$ and therefore

$$
d(a)\left[v^{*}, a\right]+a\left(d(v) a+d(v) a^{\prime}\right)=0
$$

From (2.13), replacing $u$ by $v$, we obtain $d(v) a=a d(v)$, and hence using it in (2.15), we have $d(a)\left[v^{*}, a\right]+a[d(v), a]=0$. Using (2.13) again, we have

$$
d(a)\left[v^{*}, a\right]=0
$$

Replacing $v$ by $v^{*}$, we obtain

$$
d(a)[v, a]=0
$$

In (2.17), replacing $v$ by $v u$ and using it again, we obtain $d(a) S[u, a]=0$. By the primeness of S, we have $d(a)=0$ or $[u, a]=0$ and therefore $d(a)=0$ or $a \in Z(S)$.

Theorem 2.5. Let $S$ be semiprime *-semiring. If $S$ admits a *-derivation $d$ such that $d[u, v]=0$, then $d=0$ or $S$ is commutative. 
Proof. We have for all $u, v \in S$

$$
d[u, v]=0
$$

Replacing $u$ by $u v$ in (2.18), we obtain $d[u v, v]=0$ and therefore $d[u, v] v^{*}+[u, v] d(v)=0$. Using (2.18) again, we obtain

$$
[u, v] d(v)=0
$$

Replacing $u$ by $s u$ in (2.19), we obtain $[s u, v] d(v)=0$ which implies $s[u, v] d(v)+[s, v] u d(v)=0$. Using (2.19) again, we obtain $[s, v] u d(v)=0$ and therefore

$$
[s, v] R d(v)=0
$$

By primeness of $S,(2.20)$ yields either $[s, v]=0$ or $d(v)=0$. Now take $K=\{v \in S: d(v)=0\}$ and $L=\{v \in S:[s, v]=0, \forall s \in S\}$. Clearly $S=K \cup L$. We claim that either $S=K$ or $S=L$. For this we can show that either $L \subseteq K$ or $K \subseteq L$. Suppose that $u \in K \backslash L$ and $v \in L \backslash K$. Clearly $u+v \in K+L \subseteq S=K \bigcup L$. Therefore $u+v \in K$ or $u+v \in L$. Firstly, If $u+v \in K$, then $d(u+v)=0$ which implies $d(u)+d(v)=0$ and therefore $d(v)=0$ which means $v \in K$, a contradiction. Secondly, if $u+v \in L[u+v, r]=[u, r]+[v, r]=[u, r]=0, \forall r \in S$, which implies $u \in L$, a contradiction. Therefore, we have either $L \subseteq K$ or $K \subseteq L$ and hence either $S=K$ or $S=L$. This proves that that either $d=0$ or $S$ is commutative.

Theorem 2.6. Let $S$ be prime ${ }^{*}$-semiring. If $S$ admits a ${ }^{*}$-derivation $d$ such that $d(u \circ v)=0, \forall u, v \in S$, then $d=0$ or $S$ is commutative.

Proof. For any $u, v \in S$, We have

$$
d(u \circ v)=0
$$

In (2.21) replacing $u$ by $u v$, we obtain $d((u v) \circ v)=0$. But $d((u v) \circ v)=$ $d(u \circ v) v)$. Therefore $d(u \circ v) v)=0$ and hence $d(u \circ v) v^{*}+(u \circ v) d(v)=0$. Using (2.21) again, we obtain

$$
(u \circ v) d(v)=0
$$

In (2.22) replacing $u$ by $s v$, we obtain $((s v) \circ v) d(v)=0$, which implies 


$$
(s \circ v) S d(v)=0
$$

Since $S$ is prime, therefore (2.23) yields either $(s \circ v)=0$ or $d(v)=0$. Let $K=\{v \in S: d(v)=0\}$ and $L=\{v \in S: s \circ v=0, \forall s \in S\}$. Clearly $S=K \cup L$. Our claim is that either $S=K$ or $S=L$. For this we show that either $K \subseteq L$ or $L \subseteq K$. Suppose that $u \in K \backslash L$ and $v \in L \backslash K$. Clearly $u+v \in K+L \subseteq S=K \bigcup L$, which implies $u+v \in K$ or $u+v \in L$. Firstly, If $u+v \in K$, then $d(u+v)=d(u)+d(v)=d(v)=0$ which means $v \in K$, a contradiction. Secondly, if $u+v \in L$, then $r \circ(u+v)=r \circ u+r \circ v=$ $r \circ u=0, \forall r \in S$. which means $u \in L$, a contradiction. Therefore we obtain either $L \subseteq K$ or $K \subseteq L$, which implies that either $S=K$ or $S=L$. If $S=K$, then $d=0$. On the other hand, if $S=L$, then for any $s, v \in S$

$$
s \circ v=0
$$

In (2.24) replacing $s$ by $s w$, we obtain $(s w) \circ v=0$, which implies $s w v+v s w=0$. Since $s=s+s^{\prime}+s$ and $s+s^{\prime} \in Z(S)$ therefore last equation becomes $s w v+v\left(s+s^{\prime}+s\right) w=0$ which gives on simplification that $s(w \circ v)+[v, s] w=0$. Using (2.24) again, we obtain $[v, s] w=0$. Replacing $w$ by $w u$, we obtain $[v, s] S u=0$, . By the primeness of $S$, since $S \neq 0$, we obtain $[v, s]=0$. This proves that $S$ is commutative.

Theorem 2.7. Let $S$ be prime *-semiring. If $S$ admits a ${ }^{*}$-derivation $d$ such that $d(u) \circ v=0, \forall u, v \in S$, then $d=0$ or $S$ is commutative.

Proof. We have for any $u, v \in S$

$$
d(u) \circ v=0
$$

In (2.25) replacing $u$ by $u w$, we obtain $\left(d(u) w^{*}+u d(w)\right) \circ v=0$. Since $v+v^{\prime} \in Z, v+v^{\prime}+v=v$ and $v^{\prime}+v+v^{\prime}=v^{\prime}$, after simplification we obtain

$$
(d(u) \circ v) w^{*}+d(u)\left[w^{*}, v\right]+u(v \circ d(w))+[v, u] d(w)=0
$$

Using (26), we obtain

$$
d(u)\left[w^{*}, v\right]+[v, u] d(w)=0
$$

Replacing $u$ by $v,(2.27)$, we obtain $d(v)\left[w^{*}, v\right]+[v, v] d(w)=0$. Using the definition of $S$ and simplifying we obtain 


$$
d(v)\left[w^{*}, v\right]+v\left(v d(w)+v^{\prime} d(w)\right)=0
$$

From (2.25), we have $d(w) v=v^{\prime} d(w)$. Hence (2.28) becomes $d(v)\left[w^{*}, v\right]+$ $v(d(w) \circ v)=0$. Using (2.25) again, we obtain

$$
d(v)\left[w^{*}, v\right]=0
$$

In (2.29) replacing $w$ by $w^{*}$, we obtain

$$
d(v)[w, v]=0
$$

Replacing $w$ by $u w$ in (2.30), we obtain $d(v)[u w, v]=0$ which further implies $d(v) u[w, v]+[d(u), v] w=0$. Using (2.30) again, we obtain

$$
d(v) S[w, v]=0
$$

Since $S$ is prime, therefore from $(2.31)$, we have $d(v)=0$ or $[w, v]=0$. The remaining part is same as that of Theorem 2.5.

Theorem 2.8. Let $S$ be a 2-torsion free semiprime ${ }^{*}$-semiring. Suppose that

$a u^{*} b^{*}+b u a=0, \forall u \in S$, for some $a, b \in S$. Then $a b=0=b a$. Moreover if $S$ is prime, then either $a=0$ or $b=0$.

Proof. By the hypothesis

$$
a u^{*} b^{*}+b u a=0
$$

In (2.32) replacing $u$ by $v b u$, we obtain $a(v b u)^{*} b^{*}+b u a=0$

$$
a u^{*} b^{*} v^{*} b^{*}+b v b u a=0
$$

From (2.32), using $a u^{*} b^{*}=b u a^{*}$ into (2.33), we obtain $b u a^{\prime} v^{*} b^{*}+$ bvbua $=0$ which further implies

$$
b u b v a+b v b u a=0
$$

In particular for $v=u$, we obtain 2bubua $=0, \forall u \in S$ and 2-torsion freeness of $S$ further yields

$$
\text { bubua }=0
$$

Again from (2.32), using $a u^{*} b^{*}=b u a^{*}$ into (2.35), we obtain 


$$
\text { buau }^{*} b^{*}=0
$$

In (2.35), replacing $v$ by uav, we obtain bub(uav) $a+b(u a v) b u a=0$. Using (2.32), we obtain $\left(\text { buau }^{*} b^{*}\right)^{\prime} v a+$ buavbua $=0$. Using (2.36) again, we obtain buaSbua $=0$ and therefore by the semiprimeness, we obtain

$$
b u a=0
$$

This implies $a b u a b=0$. By the semiprimeness of $S$, we have $a b=0$. Again from (2.37), we have $b a u b a=0$, which implies $b a=0$. Hence we conclude that $a b=0=b a$. Moreover if $S$ is prime then (2.37) yields either $a=0$ or $b=0$.

Theorem 2.9. Let $S$ be a 2-torsion free semiprime ${ }^{*}$-semiring and $F$ : $S \longrightarrow S$ be an additive mapping satisfying

$$
F\left(u v^{\prime} u\right)+F(u) v^{*} u^{*}+u f(v) u^{*}+u v f(u)=0, \forall u, v \in S
$$

associated with the Jordan *-derivation $f$. Then $F$ is a Jordan *-derivation.

Proof. Replacing $u$ by $u+w$ by in (2.38), we obtain

$F\left((u+w) v^{\prime}(u+w)\right)+F(u+w) v^{*}(u+w)^{*}+(u+w) f(v)(u+w)^{*}+(u+w) v f(u+w)=0$

which further implies

$F\left(u v^{\prime} u\right)+F\left(w v^{\prime} u\right)+F\left(u v^{\prime} w\right)+F\left(w v^{\prime} w\right)+F(u) v^{*} u^{*}$

$+F(u) v^{*} w^{*}+F(w) v^{*} w^{*}+F(w) v^{*} w^{*}+\left(u f(v) u^{*}\right.$

$\left.+w f(v) u^{*}\right)+u f(v) w^{*}+w f(v) w^{*}+u v f(u)+w v f(u)+u v f(w)+w v f(w)=0$.

Using (2.38) again we obtain

$F\left(w v^{\prime} u\right)+F\left(u v^{\prime} w\right)+F(u) v^{*} w^{*}$

In (2.39), replacing $w$ by $u^{2}$, we obtain

$F\left(u^{2} v^{\prime} u\right)+F\left(u v^{\prime} u^{2}\right)+F(u) v^{*} u^{* 2}+F\left(u^{2}\right) v^{*} u^{2 *}$

$$
+u^{2} f(v) u^{*}+u f(v) u^{2 *}+u^{2} v f(u)+u v f\left(u^{2}\right)=0
$$

Replacing $v$ by $u v+v u$ in (2.38), we obtain $F\left(u(u v+v u)^{\prime} u\right)+F(u)(u v+v u)^{*} u^{*}+u f(u v+v u) u^{*}+u((u v+v u)) f(u)=0$, 
which further implies

$F\left(u^{2} v^{\prime} u+u v^{\prime} u^{2}\right)+F(u) u^{*} v^{*} u^{*}+F(u) v^{*} u^{* 2}$

$\left(2+4 u f f(u) v^{*} u^{*}+u^{2} f(v) u^{*}+u f(v) u^{* 2}+u v f(u) u^{*}+u^{2} v f(u)+u v u f(u)=0\right.$

From (2.40), we have

$\left(F\left(u^{2}\right) v^{*} u^{2 *}\right)^{\prime}=F\left(u^{2} v^{\prime} u\right)+F\left(u v^{\prime} u^{2}\right)$

$$
+F(u) v^{*} u^{* 2}+u^{2} f(v) u^{*}+u f(v) u^{2 *}+u^{2} v f(u)+u v f\left(u^{2}\right)
$$

Using (2.42) into (2.41), we obtain $F(u) u^{*} v^{*} u^{*}+\left(F\left(u^{2}\right) v^{*} u^{*}\right)^{\prime}+u f(u) v^{*} u^{*}=$ 0 and therefore $F(u) u^{*^{\prime}} v^{*} u^{*}+F\left(u^{2}\right) v^{*} u^{*}+u^{\prime} f(u) v^{*} u^{*}=0$, which further implies

$$
\left(F\left(u^{2}\right)+(F(u))^{\prime} u^{*^{\prime}}+u^{\prime} f(u)\right) v^{*} u^{*}=0 S
$$

Setting $F\left(u^{2}\right)+(F(u))^{\prime} u^{*}+u^{\prime} f(u)=A(u)$ in (2.43), we obtain

$$
A(u) v^{*} u^{*}=0
$$

Replacing $v$ by $v^{*}$ in (2.44), we obtain

$$
A(u) v u^{*}=0
$$

which implies that $u^{*} A(u) R u^{*} A(u)=0$. By the semiprimeness of $S$, we obtain

$$
u^{*} A(u)=0
$$

Replacing $v$ by $u^{*} v A(u)$, we obtain $A(u) u^{*} R A(u) u^{*}=0$ and by the semiprimeness, we get

$$
A(u) u^{*}=0
$$

In (2.47) replacing $u$ by $u+v$, we obtain $A(u+v)(u+v)^{*}=0$, which further implies $(A(u)+B(u, v)+A(v))\left(u^{*}+v^{*}\right)=0$, where $B(u, v)=$ $F(u v+v u)+\left(F(u) v^{*}\right)^{\prime}+\left(F(v) u^{*}\right)^{\prime}+u^{\prime} f(v)+v^{\prime} f(u)$. Hence we have $A(u) u^{*}+B(u, v) u^{*}+A(v) u^{*}+A(u) v^{*}+B(u, v) v^{*}+A(v) v^{*}=0$. Using (2.47) again, we obtain

$$
B(u, v) u^{*}+A(v) u^{*}+A(u) v^{*}+B(u, v) v^{*}=0
$$


In (2.48) replacing $u$ by $u^{\prime}$, we obtain $B\left(u^{\prime}, v\right) u^{*^{\prime}}+A(v) u^{*^{\prime}}+A\left(u^{\prime}\right) v^{*}+$ $B\left(u^{\prime}, v\right) v^{*}=0$, which further implies $B(u, v) u^{*}+\left(A(v) u^{*}\right)^{\prime}+A(u) v^{*}+$ $\left(B(u, v) v^{*}\right)^{\prime}=0$ and hence

$$
B(u, v) u^{*}+A(u) v^{*}=A(v) u^{*}+B(u, v) v^{*}
$$

Using (2.49) into (2.48), we obtain $2\left(B(u, v) u^{*}+A(u) v^{*}\right)=0$ and by 2 -torsion freeness of $S$, we obtain

$$
B(u, v) u^{*}+A(u) v^{*}=0
$$

Multiplying (2.50) by $A(u)$ from the right, we obtain $B(u, v) u^{*} A(u)+$ $A(u) v^{*} A(u)=0$. Using (2.46), we obtain $A(u) v^{*} A(u)=0$. Replacing $v$ by $v^{*}$, we obtain $A(u) R A(u)=0$. By the semiprimeness of $S$, we obtain $A(u)=0$. Therefore $F\left(u^{2}\right)+(F(u))^{\prime} u^{*}+u^{\prime} F(u)=0$, which further implies $F\left(u^{2}\right)=F(u) u^{*}+u F(u)$ and this completes the proof.

Theorem 2.10. Let $S$ be a 2 -torsion and 3 -torsion free semiprime ${ }^{*}$-semiring and $D: S \longrightarrow S$ be an additive mapping satisfying

$$
D\left(u v^{\prime} u\right)+D(u) v^{*} u^{*}+u D(v) u^{*}+u v D(u)=0, \forall u, v \in S
$$

Then $D$ is a Jordan *-derivation.

Proof. In (2.50), replacing $u$ by $u^{2}$, we obtain

$$
D\left(u^{2} v^{\prime} u^{2}\right)+D\left(u^{2}\right) v^{*} u^{* 2}+u^{2} D(v) u^{* 2}+u^{2} v D\left(u^{2}\right)=0
$$

In (2.51) replacing $v$ by $u v u$, we obtain $D\left(u^{2} v^{\prime} u^{2}\right)+D(u) u^{*} v^{*} u^{* 2}+$ $u D(u v u) u^{*}+u(u v u) D(u)=0$. Using (2.51) into the last equation, we obtain $D\left(u^{2} v^{\prime} u^{2}\right)+D(u) u^{*} v^{*} u^{* 2}+u\left(D(u) v^{*} u^{*}+u D(v) u^{*}+u v D(u)\right) u^{*}+$ $u(u v u) D(u)=0$. Therefore

$D\left(u^{2} v^{\prime} u^{2}\right)+D(u) u^{*} v^{*} u^{* 2}+u D(u) v^{*} u^{* 2}+u^{2} D(v) u^{* 2}+u^{2} v D(u) u^{*}+u^{2} v u D(u)=0$

Since $v+v^{\prime} \in Z, v+v^{\prime}+v=v, v^{\prime}+v+v^{\prime}=v^{\prime}$, therefore from (2.52) , we have

$$
D\left(u^{2} v^{\prime} u^{2}\right)+u^{2} D(v) u^{* 2}=D\left(u^{2}\right) v^{*^{\prime}} u^{* 2}+u^{2^{\prime}} v D\left(u^{2}\right.
$$


Using (2.54) into (2.53), we obtain $D(u) u^{*} v^{*} u^{* 2}+u D(u) v^{*} u^{* 2}+u^{2} v D(u) u^{*}+$ $u^{2} v u D(u)+D\left(u^{2}\right) v^{*^{\prime}} u^{* 2}+u^{2^{\prime}} v D\left(u^{2}=0\right.$. This further implies $u^{2} v^{\prime}\left(D\left(u^{2}+\right.\right.$ $\left.D(u) u^{*^{\prime}}+u^{\prime} D(u)\right)+\left(D\left(u^{2}\right)+D(u) u^{*^{\prime}}+u^{\prime} D(u)\right) v^{*^{\prime}} u^{* 2}=0$ and therefore $u^{2} v\left(D\left(u^{2}+D(u) u^{*^{\prime}}+u^{\prime} D(u)\right)+\left(D\left(u^{2}\right)+D(u) u^{*^{\prime}}+u^{\prime} D(u)\right) v^{*} u^{* 2}=0\right.$. Setting $A(u)=D\left(u^{2}+D(u) u^{*^{\prime}}+u^{\prime} D(u)\right.$ into the last equation, we obtain

$$
u^{2} v A(u)+A(u) v^{*} u^{* 2}=0
$$

In view of Theorem 2.8 , we can write

$$
\begin{aligned}
& A(u) u^{2}=0 \\
& u^{2} A(u)=0
\end{aligned}
$$

linearizing (2.56), we obtain

$$
A(u+v)(u+v)^{2}=0
$$

We can easily see that $A(u+v)=A(u)+B(u, v)+A(v)$, where $B(u, v)=$ $D(u v+v u)+(D(u))^{\prime} v^{*}+(D(v))^{\prime} u^{*}+u^{\prime} D(v)+v^{\prime} D(u)$. Hence (59) becomes $A(u) u^{2}+B(u, v) u^{2}+A(v) u^{2}+A(u) v^{2}+B(u, v) v^{2}+A(v) v^{2}+A(u)(u v+v u)$ $+B(u, v)(u v+v u)+A(v)(u v+v u)=0$. Using (2.56) again in the last equation, we obtain

$B(u, v) u^{2}+A(v) u^{2}+A(u) v^{2}+B(u, v) v^{2}+A(u)(u v+v u)$

$$
+B(u, v)(u v+v u)+A(v)(u v+v u)=0
$$

We can easily observe that $A\left(u^{\prime}\right)=A(u)$ and $B\left(u^{\prime}, v\right)=(B(u, v))^{\prime}$. Replacing $u$ by $u^{\prime}$ in (2.59), we obtain $(B(u, v))^{\prime} u^{2}+A(v) u^{2}+A(u) v^{2}+(B(u, v))^{\prime} v^{2}+A(u)(u v+v u)^{\prime}$

$$
+B(u, v)(u v+v u)+A(v)(u v+v u)^{\prime}=0
$$

From (2.60), we have $A(v) u^{2}+A(u) v^{2}+B(u, v)(u v+v u)$

$(2.61)=(B(u, v)) u^{2}+(B(u, v)) v^{2}+A(u)(u v+v u)+A(v)(u v+v u)$

Using (2.61) into (2.59), we obtain $2\left((B(u, v)) u^{2}+(B(u, v)) v^{2}+A(u)(u v+\right.$ $v u)+A(v)(u v+v u))=0$. Since $S$ is 2-torsion free, therefore 


$$
\left.B(u, v) u^{2}+B(u, v) v^{2}+A(u)(u v+v u)+A(v)(u v+v u)\right)=0
$$

We can easily see that $A(2 u)=4 A(u)$ and $B(2 u, v)=2 B(u, v)$. Replacing $u$ by $2 u$ in (2.62), we obtain $8 B(u, v) u^{2}+2 B(u, v) v^{2}+8 A(u)(u v+$ $v u)+2 A(v)(u v+v u)=0$, which can also be written as $2\left(4 B(u, v) u^{2}+\right.$ $\left.B(u, v) v^{2}+4 A(u)(u v+v u)+A(v)(u v+v u)\right)=0$. By the 2-torsion freeness of $S$, we obtain

$(2.63) 4 B(u, v) u^{2}+B(u, v) v^{2}+4 A(u)(u v+v u)+A(v)(u v+v u)=0$

Since Since $v+v^{\prime} \in Z, v+v^{\prime}+v=v, v^{\prime}+v+v^{\prime}=v^{\prime}$, therefore from (2.62), we have

$\left.(2.64) B(u, v) v^{2}+A(v)(u v+v u)\right)=(B(u, v))^{\prime} u^{2}+(A(u))^{\prime}(u v+v u)$

Using (2.64) into (2.63), we obtain $4 B(u, v) u^{2}+(B(u, v))^{\prime} u^{2}+4 A(u)(u v+$ $v u)+(A(u))^{\prime}(u v+v u)=0$. Since $u+u^{\prime}+u=u, u^{\prime}+u+u^{\prime}=u^{\prime}$, therefore $3 B(u, v) u^{2}+3 A(u)(u v+v u)=0$ and hence by 3 -torsion freeness of $S$, we have

$$
B(u, v) u^{2}+A(u)(u v+v u)=0
$$

Multiplying (2.65) by $A(u) u$ from the right and using (2.57), we obtain

$$
A(u) u v A(u) u+A(u) v u A(u) u=0
$$

In (2.66), replacing $v$ by $v u$, we obtain $A(u) u v u A(u) u+A(u) v u^{2} A(u) u=$ 0 . Using (2.56), we obtain $A(u) u v u A(u) u=0$, which further implies $u A(u) u R u A(u) u=0$. By the semiprimeness of $S$, we obtain

$$
A(u) u=0
$$

Hence (2.65) becomes

$$
B(u, v) u^{2}+A(u) v u=0
$$

Multiplying (2.68) by $A(u)$ from the right and using (2.57), we obtain $A(u) v u A(u)=0$, which implies $u A(u) v u A(u)=0$ and hence $u A(u) R u A(u)=$ 0 and by the semiprimeness, we have 


$$
u A(u)=0
$$

From (2.68), we have $(B(u, v) u+A(u) v) u=0$, which implies $(B(u, v) u+$ $A(u) v) u(B(u, v) u+A(u) v)=0$ and therefore

$$
B(u, v) u+A(u) v=0
$$

Multiplying (2.70) by $A(u)$ from the right, we obtain $B(u, v) u A(u)+$ $A(u) v A(u)=0$ and using (2.70) again, we obtain $A(u) v A(u)=0$. Since $S$ is semiprime, therefore $A(u)=0$. This means $D\left(u^{2}\right)+D(u) u^{*^{\prime}}+u^{\prime} D(u)=0$ and hence $D\left(u^{2}\right)=D(u) u^{*}+u D(u)$, which shows that $D$ is Jordan *derivation.

\section{Concluding Remarks}

This article presents some criteria for *-derivations which induce commutativity in additive inverse semirings with involution. Secondly we present some additive mappings satisfying certain conditions under which they become Jordan *-derivations. Therefore ideas presented in this article are useful. We propose some open problems as follows:

1. Let $S$ be a semiprime ${ }^{*}$-semiring and $d$ a nonzero ${ }^{*}$-derivation of $S$ satisfying $d(u) \circ v=0, \forall u, v \in S$. Is $S$ commutative?

2. Let $S$ be a semiprime *-semiring and $d$ a nonzero *-derivation of $S$ satisfying $d(u \circ v)=0, \forall u, v \in S$. Is $S$ commutative?

3. Let $S$ be a prime *-semiring, $d$ a nonzero *-derivation of $S$ and $F$ an additive mapping defined by $F(x y)=F(x) y+x d(y)$. If $F$ satisfies $F(u \circ v)=0, \forall u, v \in S$. Is $S$ commutative?

\section{References}

[1] L. Ali, M. Aslam, and Y. A. Khan, "Commutativity of semirings with involution", Asian-European journal of mathematics, Art ID. 2050153, 2019, doi: 10.1142/S1793557120501533

[2] L. Ali, M. Aslam, and Y. A. Khan, "On Jordan ideals of inverse semirings with involution", Indian journal of science and technology, vol. 13, no, 4, pp. 430-438, 2020- [On line]. Available: https://bit.ly/2ZrljRb 
[3] L. Ali, M. Aslam, and Y. A. Khan, "Some commutativity conditions on *-prime semirings", JP journal of algebra, number theory and applications, vol. 46, no. 2, pp. 109-121, May 2020, doi: 10.17654/NT046020109

[4] L. Ali, M. Aslam, and Y. A. Khan, "On generalized derivations of semirings with involution", Journal of mechanics continua and mathematical sciences, vol. 15, no. 4, Apr. 2020, doi: $10.26782 / \mathrm{jmcms} .2020 .04 .00011$

[5] K. I. Beidar and W. S. Martindale, "On functional identities in prime rings with involution", Journal of algebra, vol. 203, no. 2, pp. 491-532, May 1998, doi: 10.1006/jabr.1997.7285

[6] M. Brešar and J. Vukman, "On some additive mappings in rings with involution", Aequationes mathematics, vol. 38, no. 2-3, pp. 178-185, Jun. 1989, doi: 10.1007/BF01840003

[7] I. N. Herstein, Rings with involution, Chicago, IL: University of Chicago, 1976.

[8] M. A. Javed, M. Aslam, and M. Hussain, "On condition (A2) of Bandlet and Petrich for inverse semirings", International mathematical forum, vol. 7, no. 59, pp. 2903-2914, 2012. [On line]. Available: https://bit.ly/3fqASOw

[9] Y. A. Khan, M. Aslam, and L. Ali, "Commutativity of additive inverse semirings through $\mathrm{f}(\mathrm{xy})=[\mathrm{x}, \mathrm{f}(\mathrm{y})]$ ", Thai journal of mathematics, special issue, pp. 288-300, 2018. [On line]. Available: https://bit.ly/38RgRhz

[10] K. H. Kim and Y. H. Lee, "A note on *-derivation of *-prime rings", International mathematical forum, vol. 12, no. 8, pp. 391-398, 2017, doi: 10.12988/imf.2017.7114

[11] C. Lanski, "Commutation with skew elements in rings with involution", Pacific journal of mathematics, vol. 83, no. 2, pp. 393-399, Aug. 1979, doi: $10.2140 /$ pjm.1979.83.393

[12] T. K. Lee and P. Y. Shen, "On derivations of prime rings with involution", Chinese journal of mathematics, vol. 20, no. 2, pp. 191-203, Jun. 1992. [On line]. Available: https://bit.ly/2ZnI4oP

[13] L. Oukhtite and S. Salhi, "On derivations in $\sigma$-prime rings", International journal of algebra, vol. 1, no. 5, pp. 241-246, 2007, doi: 10.12988/ija.2007.07025 
[14] T. W. Palmer, Banach algebras and the general theory of *algebras, vol. 2. Cambridge: Cambridge University Press, 2001, doi: 10.1017/CBO9780511574757

[15] S. Sara, M. Aslam, and M. A Javed, "On centralizer of semiprime inverse semiring", Discussiones mathematicae - General algebra and applications, vol. 36, no.1, pp. 71-84, 2016, doi: 10.7151/dmgaa.1252

[16] I. E. Segal, "Irreducible representations of operator algebras", Bulletin American Mathematics and Society, vol. 53, pp. 73-88, Feb. 1947, doi: 10.1090/S0002-9904-1947-08742-5 